\title{
Office-Based Steroid Injection in Benign Vocal Fold Lesions
}

\section{Original Article}

Baraka, M. ', Essam Abd-elwanees Behairy², Hossam El Desouky33, Shaimaa Mostafa ${ }^{4}$,

\author{
Ezzat,.$^{5}$
}

Department of Otorhinolaryngology, Faculty of Medicine, 'Ain Shams University, Cairo,

${ }^{2,5}$ El Menoufia University, Menoufya, ${ }^{3}$ Cairo University, Cairo, ${ }^{4}$ Phoniatric Department,

Hearing and Speech Institute.

\begin{abstract}
Background: Benign vocal fold lesions (BVFLs) are usually caused by vocal abuse or misuse. Conservative treatment and micro laryngeal surgery (MLS) are recommended in the treatment. However, conservative measures are usually difficult to perform and some patients are contraindicated for general anesthesia.

Objective: This study is designed to assess the practical application, effectiveness, and side effects of office-based steriod injection via a transnasal route.

Patients and Methods: This study included 29 patients with BVFLs. Transnasal endoscopic steroid injection (TESI) was performed via operating channel for all patients. Local anesthesia was used to inject betamethasone into Reinke's space at the base of the lesion. Acoustic analysis, including fundamental frequency (F0), jitter, shimmer, noise to a harmonic ratio (NHR), aerodynamic analysis (MPT), auditory perceptual assessment using modified GRBAS scale, videolaryngostroboscopy (VLS) evaluations, and voice handicap index (VHI), was done before and three months after TESI.

Results: Three months after injection, the VLS demonstrated that remission in size or lesions improvement was observed in 20 patients, total disappearance in 4 patients, and no improvement in 5 patients. The summation of the modified GRBAS scale also indicated highly significant improvement after TESI $(P<0.01)$. MPT showed a highly significant increase post-injection. VHI showed a highly significant reduction post-injection. Acoustic analysis revealed significant improvements in F0, jitter, shimmer, and NHR $(P<0.05)$.
\end{abstract}

Conclusion: : Office-based TESI is an effective, safe, easy, and feasible alternative treatment method in some BVFLs.

Key Words: Benign vocal fold lesions, intralesional injection, steroids, transnasal endoscopic injection.

Received: 19 July 2021, Accepted: 24 August 2021

Corresponding Author: Shaimaa Mostafa, Phoniatric Department, Hearing and Speech Institute, Egypt, Tel.: +201010101807, E-mail: : shaimaad@yahoo.com

ISSN: 2090-0740, 2021

\section{INTRODUCTION}

Among benign vocal fold lesions (BVFLs), minimal associated pathological lesions, including vocal fold (VF) polyp, nodules, Reinke's edema, cyst, and vocal process granuloma, represent a distinct clinical and pathological entity. Pathophysiologically, they usually occur due to acute or chronic voice forcing (phonotrauma) and usually have the same predisposing factors as smoking, or inhaled irritants $^{[1]}$.

Such lesions are common, especially among professional voice users, and can affect their quality of life. These lesions cause dysphonia, narrowed vocal range, and deteriorated voice quality, so selecting proper and effective treatment is important ${ }^{[2]}$.

Conservative measures including voice rest and regular attendance of voice therapy sessions remain the first lines of treatment. However, compliance with voice therapy is usually poor, impairing treatment outcomes in the majority of cases $^{[3]}$.

When behavioral measures fail or unsuitable for some patients, micro laryngeal surgery (MLS) is usually recommended. However, there is a risk of iatrogenic complications as dental trauma, cervical spine trauma, lingual paresthesia or anesthesia, and iatrogenic VF scarring, in addition to the risks of general anesthesia ${ }^{[4]}$.

With the move toward non-invasive therapy and advancements in endoscopic imaging and instruments, it became possible to offer the VFs local steroid injection as an alternative to conventional surgery, bridging the therapeutic gap between conservative treatment and conventional MLS ${ }^{[5]}$. 
Steroids are molecules synthesized by our bodies. They regulate the immune and metabolic processes. Glucocorticoids are the most effective anti-inflammatory agent available and can be administrated via systemic or local routes. Systemic use of steroids carries a potential risk for adverse side effects in many organs depending on their duration of administration ${ }^{[6]}$.

In comparison to systemic administration, intra lesional steroid injection (ILSI) achieves a greater primary site concentration and has a lower risk of side effects. Corticosteroids act by inhibiting both collagen deposition and collagen synthesis caused by transforming growth factor-beta (TGF-beta). Also, it acts by inhibiting fibroblastic proliferation and activity plus reduction of the extracellular matrix deposition ${ }^{[5]}$.

Yanagihara et al. started VFSI in 1964. The 1 st technique had pitfalls, but with the recent advancement of endoscopy and the appearance of more high-definition pictures, many studies reported improvement in the technique ${ }^{[7,8]}$.

Office-based vocal fold steroid injection (VFSI) can be performed through transoral, percutaneous and transnasal routes. Transoral and percutaneous injection methods have many limitations. Transoral steroid injection requires passing a long, curved needle along the sensitive tongue base and pharyngeal mucosa, which can elicit the gag reflex more easily and interfere with the procedure. The percutaneous route of injection is more invasive as it needs passing of the needle through the laryngeal mucosa and inserting the needle blindly in the undersurface of the VF without directly visualizing the needle tip, which necessitates significantly higher surgical skills for proper precision $^{[9]}$.

To overcome these restrictions, the injection approach was modified utilizing the operational channel of the transnasal flexible laryngoscope, which is a more practical and technically simple procedure with good precision ${ }^{[9]}$.

VFSI has several distinct benefits. Compared to MLS, it may be conducted in the office under local anesthesia in about 20 minutes, saving high costs of general anesthesia and hospital admission. The treatment outcomes often manifest after one to two weeks of VFSI, decreasing lost workdays associated with recurrent voice therapy sessions. VFSI has a low rate of morbidity and is repeatable. Failure in VFSI has few restrictions on subsequent therapy options, and VFSI is not constrained by anatomical differences ${ }^{[10]}$.

In recent studies, the rates of recurrence declined due to advances in techniques of injection and injectable agents. Wang et al. (2013) reported few complications of $\mathrm{VFSI}^{[11]}$. One complication is the VF hematoma which subsides within one week. Also, vocal atrophy can occur with deep steroid injection in the vocalis muscle, so a superficial injection of a small amount of steroid in the Reinke's space should be confirmed. One of the common complications is whitish deposition on the VFs when injecting triamcinolone that occurs due to deposition of the chalky material of the deposit, in addition, deposition of triamcinolone in the needle resulting in its obstruction due to high viscosity of the material. To overcome this complication, the steroid regimen was modified by triamcinolone and dexamethasone mixing by half (50:50) or using dexamethasone only, which is preferable in transnasal endoscopic steroid injection (TESI), as it can pass easily without deposition.

This study aims to prove the effectiveness of officebased ILSI as an alternative modality for the treatment of BVFLs to choose the best method of treating these cases.

\section{PATIENTS AND METHODS}

This study is a prospective multicenter study carried out between December 2017 and December 2019 at Al-Menoufia University Hospital, Ain Shams University Hospital, and Hearing and speech institute. An office-based TESI of BVFLs study was applied on 29 patients with BVFLs, including VF (polyps, nodules, cysts, Reinke's edema, and vocal process granulomas). Some of them are high occupational vocal demands, and the others were of ordinary occupational vocal demands. Patients recruited from the out-patient clinics complained of dysphonia and aged between 18 and 70 years. The study goal and technique were described to patients who provided written informed consent in the appropriate language, including the study title, purpose, procedures, benefits, risks, right to reject, and signature. The university ethics committee approved the study.

The study included adult patients with small or medium-sized sessile BVFLs who refused voice therapy or were contraindicated for MLS.

The study excluded patients with large, pedunculated, and obstructing lesions, those responding to voice therapy or having other causes of dysphonia, including precancerous and cancerous lesions, and in case of structural and functional VF abnormalities accompanying the BVFLs such as VF paralysis, scarring, vocal atrophy, and sulcus vocalis. The study also excluded patients with a severe bleeding tendency or coagulopathy disorder.

\subsection{Primary assessment}

Primary assessment before injection was done, and the patients underwent the following:

2.1.1 History taking (age, gender, occupation, and symptoms duration).

\subsubsection{Subjective assessment}

- Clinical examination by videolaryngostroboscopy (VLS) (KAY PENTAX USA system) to assess the appearance of the VF edge and the vibratory property of the mucosa. The type of lesion was detected based on the 
published nomenclature and diagnostic paradigm. Size of lesion was categorized into five groups $(0=$ normal $)$, $(1=<2 \mathrm{~mm}),(2=2-4 \mathrm{~mm}),(3=4-6 \mathrm{~mm})$, and $(4=>6 \mathrm{~mm})$. Glottal closure (GC) also was assessed and categorized into four groups $(0=$ large gap $>2 \mathrm{~mm}),(1=$ moderate gap $=1-2 \mathrm{~mm}),(2=$ mild gap $<1 \mathrm{~mm})$, and $(3=$ no gap $)$. Each VLS test session was recorded digitally.

- A voice capacity self-assessment questionnaire using the Arabic version of VHI was used to assess the patients' voices self-perception.

- A high-fidelity voice record for each patient was performed. The APA of each record was performed using a modified GRBAS scale (Grade of dysphonia, Roughness, Breathiness, Asthenia, and Straining), and the grade of dysphonia was categorized by dysphonia severity index $(0=$ Normal, $1=$ Mild deviation, $2=$ Moderate deviation, 3 $=$ Severe deviation $)^{[12]}$.

\subsubsection{Objective measures:}

- Acoustic voice analysis was performed by asking the patient to phonate and recording a 5-second sample of the sustained vowel /a/ at a comfortable pitch and volume level with a distance of approximately $15-20 \mathrm{~cm}$ between the microphone and the mouth. Samples were directly recorded onto the computer using a condenser microphone and CSL software (Kay Pentax, USA), and Fo, jitter, shimmer, and NHR were automatically analyzed.

- Maximum phonation time (MPT) (an aerodynamic measure) was determined in similar conditions by using CSL software (Kay Pentax, USA). the patient was directed to produce a sustained /a/ vowel for as long as possible following deep inhalation and at a comfortable pitch and volume level for three subsequent trials.

\subsection{Procedure:}

\subsubsection{Injection technique:}

At the out-patient clinic, TESI was performed, with the patient seated on an examination chair. Before injection, local anesthesia was introduced in the nasal cavity using two sprays of Xylocaine $10 \%$ solution and a cotton pledget soaked in the nasal cavity with 1:1,000 epinephrine and $2 \%$ lidocaine. Another spray was applied in the oral cavity, over the pharynx, tonsils, and vallecula.

With the cooperation of two specialists and while the patient was seated comfortably, the injection was performed by using a non-disposable flexible needle tract with a 27 -gauge rigid tip passed through the side channel of the flexible laryngoscope (Fiber Laryngoscope FLT-III Therapeutic type) connected to a high-definition video processor. The needle tract was filled with $0.5-1$ $\mathrm{mL}$ betamethasone, which was injected into the Reinke's space just beneath the base of the lesion (in cases of polyps, nodules, and granuloma) or inside the lesions (in cases of Reinke's edema and cysts). A subepithelial transparent bleb development over Reinke's space allowed for precise positioning of the needle tip.
Adequate hydration and relative voice rest for three days, including minimal talking and avoidance of straining activities to prevent leakage of the injected material, were required as postoperative care. Additionally, patients administered proton pump inhibitors for two weeks following surgery to avoid a delay in recovery due to laryngeal irritation caused by reflux ${ }^{[13]}$.

\subsection{Secondary Assessment:}

Secondary assessment using all the subjective and objective measures was done three months post-injection.

The degree of improvement in the size of the lesions among the studied cases was recategorized as 1) complete VF lesions resolution, 2) much improvement (more than $50 \%$ reduction of lesion size), 3) some improvement (less than $50 \%$ of lesion size reduction), and 4 ) no change in lesion size.

The high-fidelity voices recorded and video files of VLS were re-evaluated by two phoniatricians with experience of more than 20 years in a blinded fashion. They knew that the files were related to VFSI, but they did not know what time they were examined or the initial diagnosis. Intra-judge reliability for each clinician and the inter-judge agreement between them for dysphonia and VLS findings (according to the size of the lesion and according to the glottal gap) were reported.

\subsection{Statistical analysis:}

Data were collected, revised, coded, and entered into the Statistical Package for Social Science (IBM SPSS) version 23. The quantitative data were presented as mean, standard deviations, and ranges when their distribution was found parametric, while qualitative data were presented as numbers and percentages.

The comparison between two independent groups with qualitative data was made using the Chi-square test and/or Fisher exact test only when the expected count in any cell was found less than 5 .

The comparison between two independent groups with quantitative data and parametric distribution was made using paired t-test, while non-parametric distribution was done using the Wilcoxon test.

The confidence interval was set to $95 \%$, and the margin of error accepted was set to $5 \%$. So, $P$-values $<0.05$ were considered significant, values, $P<0.01$ were considered highly significant, and $P$-values $>0.05$ were considered non-significant.

\section{RESULTS}

The study included 29 patients, 12 females (41.4\%) and 17 males $(58.6 \%)$. The mean age was $37.55 \pm 12.81$, ranging from 18-70 years. Eight patients $(27.6 \%)$ underwent a single injection, and 21 patients $(72.4 \%)$ underwent two or more injections, with a two-week interval in case of 
polyp, nodules, cyst, and Reinke's edema and one-month interval in granuloma according to the size of the lesion. The maximum number of injections was four times in the case of granuloma.
The distribution of different types of BVFLs among the studied cases (figure 1) were $11 \mathrm{VF}$ polyps $(37.9 \%), 7 \mathrm{VF}$ cysts $(24.1 \%), 5$ VF nodules $(17.2 \%), 4$ Reinke's edema $(13.8 \%)$, and two vocal process granulomas $(6.9 \%)$.

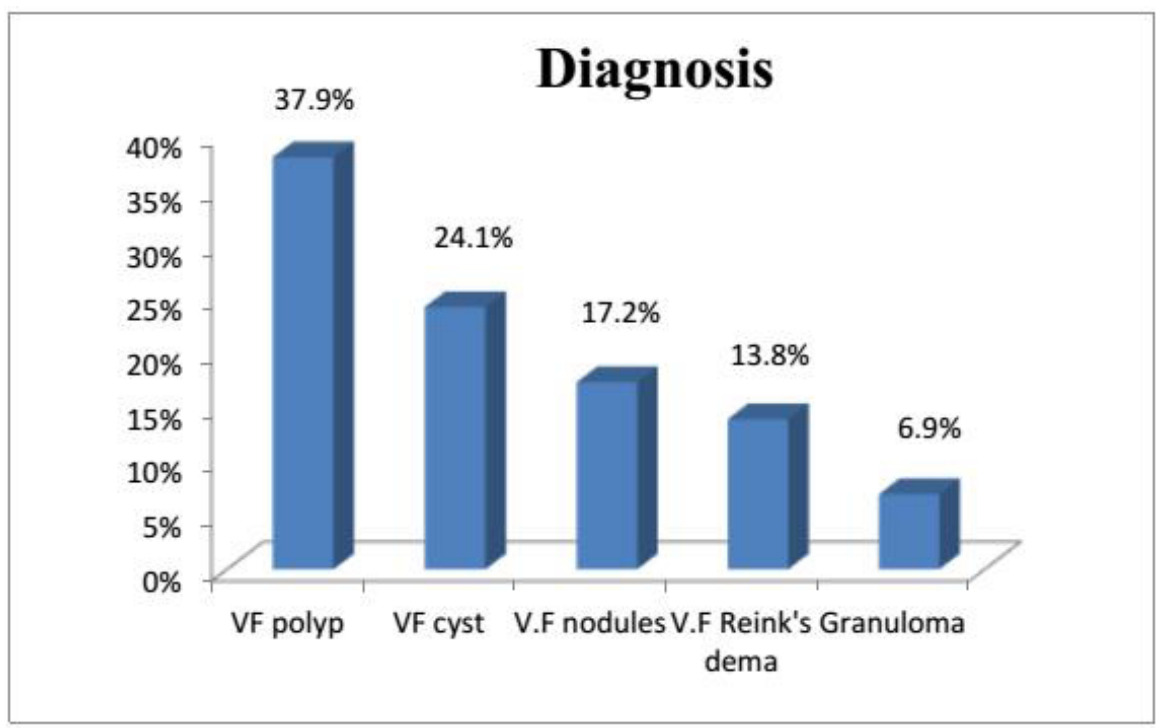

Fig. 1: This figure demonstrates the distribution of different types of BVFLs in the study.

VLS results (as regard the size of the lesions) (Table 1) showed highly significant improvement $(P$-value $<0.01)$. As regards the degree of improvement in the size of lesions among the studied cases demonstrated in Figure 2, 17.2\% of the cases showed some improvement (less than 50\% reduction of lesion size) (Figure 3), 51.7\% of the cases showed much improvement (greater than 50\% reduction of lesion size) (Figure 4), the lesions completely disappeared in $13.8 \%$ of the cases (Figure 5). On the other hand, $17.2 \%$ of the cases showed no improvement.

VLS results (according to Gc) (table 1) showed significant improvement post-ILSI (P-value $<0.05$ ), most of the cases were of grade 1 (moderate gap 37.9\%) preILSI. Post-ILSI, most of the cases became grade 2 (mild gap $44.8 \%$ ) and grade 3 (No gap $44.8 \%$ ).

Table 1: The improvement of the size of the lesion and glottic closure pre and post ILSI

\begin{tabular}{|c|c|c|c|c|c|}
\hline \multirow{2}{*}{ VLS (size of lesion) } & & Pre & Post & \multirow{2}{*}{$\mathrm{X}^{2}$} & \multirow{2}{*}{$P$} \\
\hline & & No. $(\%)$ & No. $(\%)$ & & \\
\hline 0 (No lesion) & & $0(0.0 \%)$ & $4(13.8 \%)$ & \multirow{5}{*}{$34.667 *$} & \multirow{5}{*}{0.000} \\
\hline $\mathrm{I}<2 \mathrm{ml}$ & & $4(13.8 \%)$ & $20(69.0 \%)$ & & \\
\hline II 2-4 ml & & $6(20.7 \%)$ & $0(0.0 \%)$ & & \\
\hline III 4-6 ml & & $14(48.3 \%)$ & $0(0.0 \%)$ & & \\
\hline IV $>6 \mathrm{ml}$ & & $5(17.2 \%)$ & $5(17.2 \%)$ & & \\
\hline \multirow{4}{*}{$\mathrm{GC}$} & $0=$ large $>2 \mathrm{ml}$ & $2(6.9 \%)$ & $1(3.4 \%)$ & \multirow{4}{*}{9.534} & \multirow{4}{*}{0.023} \\
\hline & $1=$ moderate $1-2 \mathrm{ml}$ & $11(37.9 \%)$ & $2(6.9 \%)$ & & \\
\hline & $2=$ mild $<1 \mathrm{ml}$ & $10(34.5 \%)$ & $13(44.8 \%)$ & & \\
\hline & $3=$ no gap & $6(20.7 \%)$ & $13(44.8 \%)$ & & \\
\hline
\end{tabular}




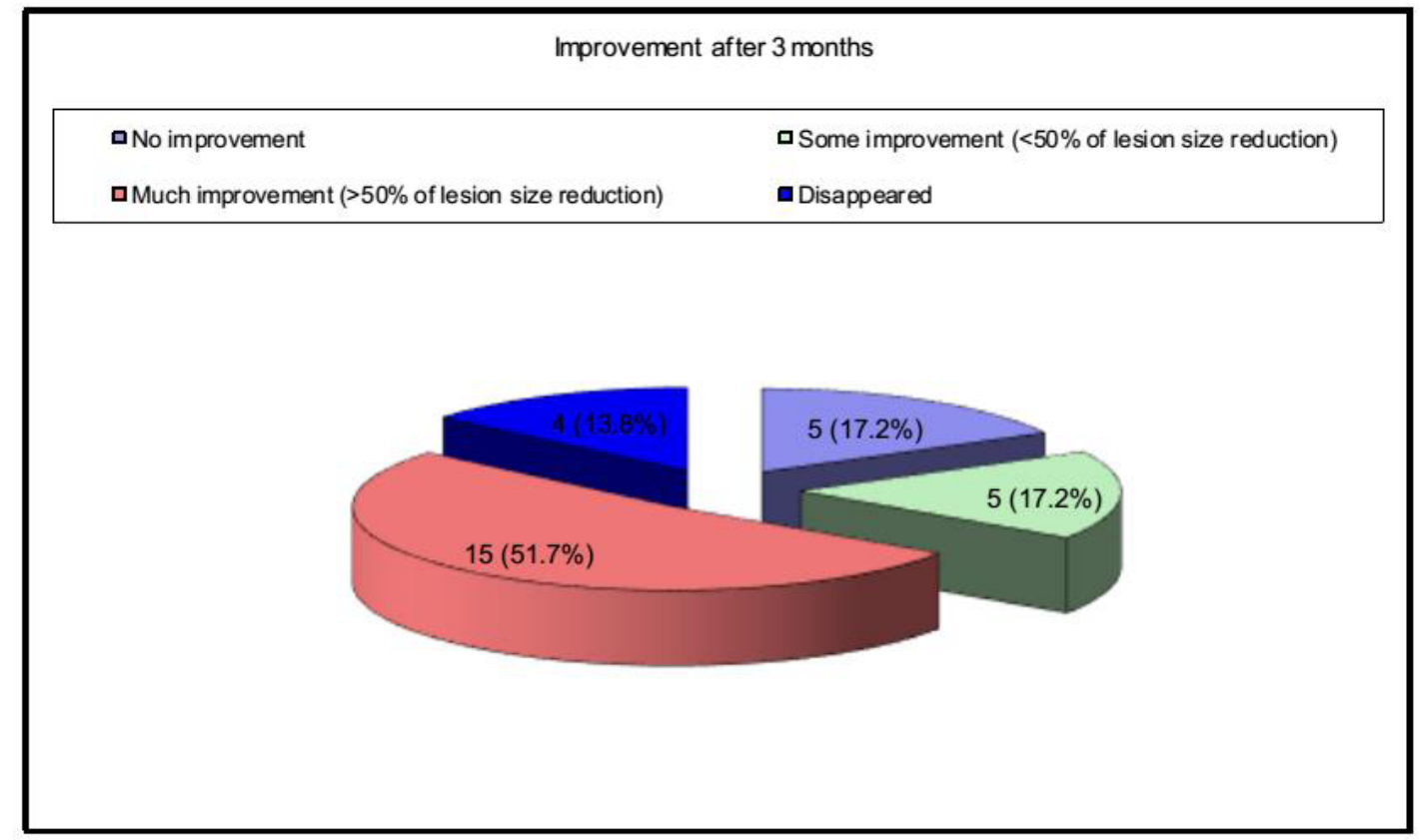

Fig. 2: This figure demonstrates the degree of improvement in the size of lesions among the studied cases.

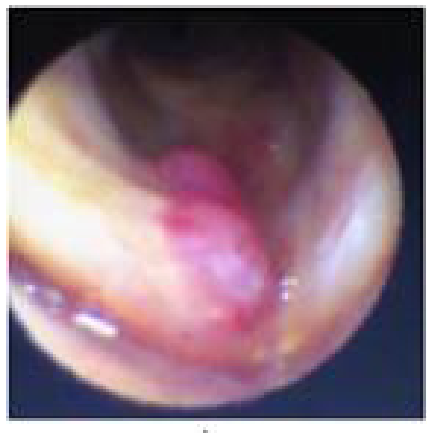

A

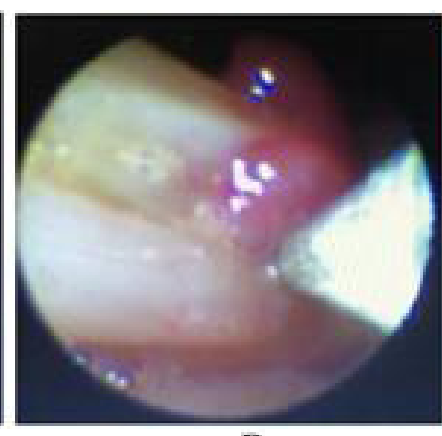

B

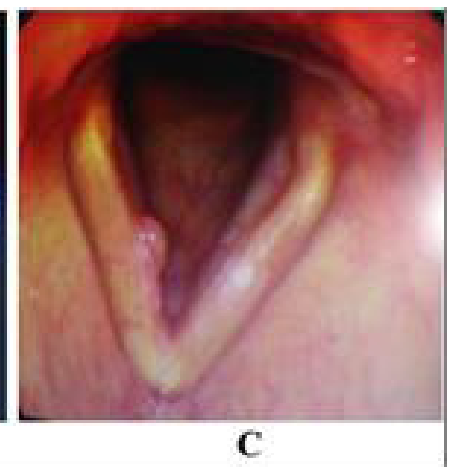

C

Fig. 3: Male patient 42 years old, with lobulated right VF polyp, he has a history of cardiomyopathy and contraindicated for general anesthesia. TESI injections were done (A), the lobulated polyp pre-injection (B) during the injection (C) 2weeks month after injection.

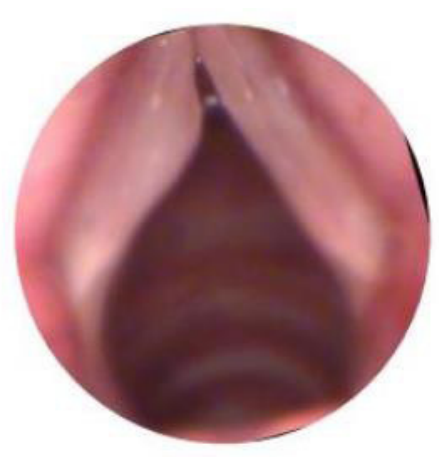

A

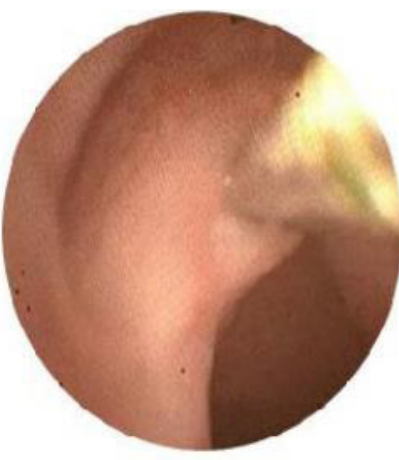

B

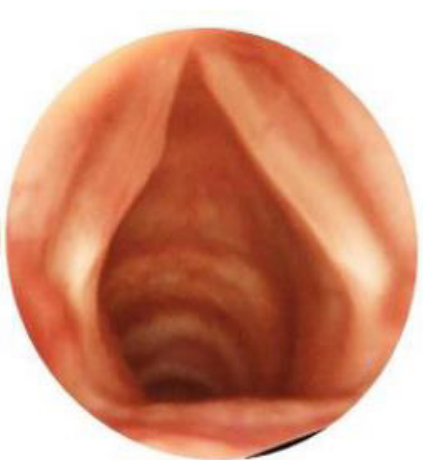

C

Fig. 4: Left VF cyst, TESI was done (A) left vocal cyst pre-injection, (B), during injection, (C) 2 weeks post-injection. 


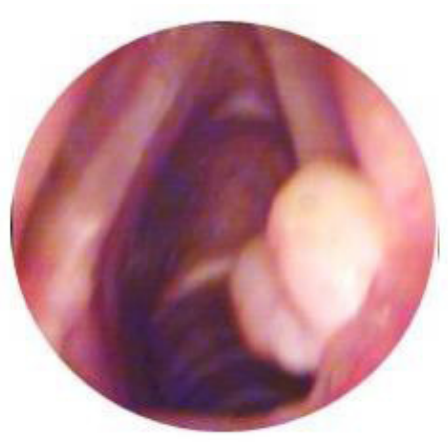

A

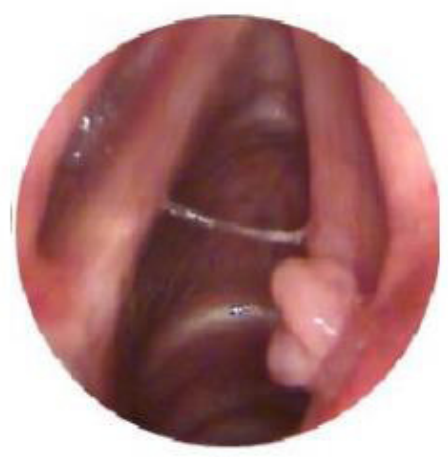

C

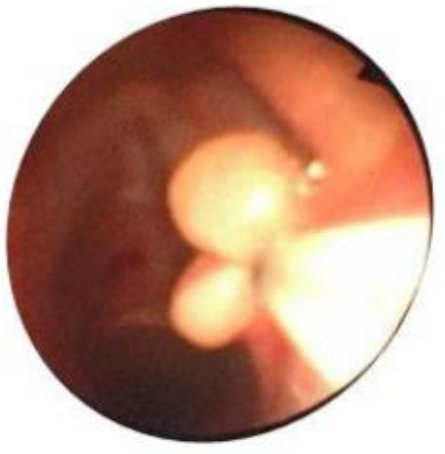

B

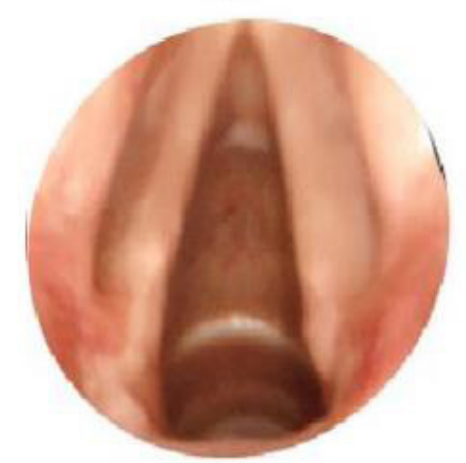

$\mathrm{D}$

Fig. 5: Recurrent right vocal process granuloma after one year from MLS, the patient received four successive TESI one month apart (A) vocal process granuloma pre-injection, (B) vocal process granuloma during the second injection (C) improvement of the vocal process granuloma after the 3rd injection (D) complete disappearance of the vocal process granuloma after the 4th injection.

Grade of dysphonia (Table 2) showed highly significant improvement $(P$-value $<0.01)$ severe degree improved from $(69.0 \%)$ to $(13.8 \%)$ and $(13.8 \%)$ of the patient became of normal voice post-ILSI compared to $(3.4 \%)$ pre-ILSI.

The GRBAS scale was used to assess perceived voice quality and revealed significantly significant improvements $(P$-value $<0.01)$. When GRBAS data were summed following ILSI, the mean scale decreased from 7.72 preILSI to 5.69 post-ILSI (Table 3).

MPT measurements (Table 3), showed highly significant improvement from 10.24 seconds before treatment to 12.73 seconds post-treatment $(P$-value $<0.001)$.

VHI assessments (table 3 ) showed a highly significant reduction from 91.38 points (severe) before ILSI to 53.45 points (moderate) post ILSI $(P$-value $<0.01)$.

Acoustic study (table 4) revealed a statistically significant improvement in Fo, jitter percent, shimmer percent, $(P$-value $<0.05)$, their means were $(183.21 \mathrm{~Hz}$, $2.63 \%$ and $4.74 \%$ ) post ILSI compared to $(173.2 \mathrm{~Hz}$, $2.98 \%$ and $5.18 \%$ ) pre ILSI respectively, NHR showed significant reduction post ILSI $(P$-value $<0.05)$, its mean decreased from 0.20 pre ILSI to 0.19 post ILSI.

Table 2: The improvement in grades of dysphonia pre and post ILSI

\begin{tabular}{lccc}
\hline \multirow{2}{*}{ Dysphonia } & Pre & Post & $X^{2}$ \\
\cline { 2 - 3 } & No. (\%) & No. $(\%)$ & \\
\hline Normal (0) & $1(3.4 \%)$ & $4(13.8 \%)$ & \\
Slight (1) & $3(10.3 \%)$ & $11(37.9 \%)$ & $18.705^{*}$ \\
Moderate (2) & $5(17.2 \%)$ & $4(13.8 \%)$ & 0.000 \\
Severe (3) & $20(69.0 \%)$ & $4.5 \%)$ & \\
\hline
\end{tabular}


Table 3: The improvement in summation of GRBAS scale, VHI, and MPT pre and post ILSI

\begin{tabular}{lccc}
\hline & Pre & Post & T \\
\hline VHI & & & \\
Mean \pm SD & $91.38 \pm 19.22$ & $53.45 \pm 23.65$ & $10.988 \bullet$ \\
Range & $50-120$ & $20-110$ & 0.000 \\
MPT & & & $-17.430 \bullet$ \\
Mean \pm SD & $10.24 \pm 1.17$ & $12.73 \pm 1.46$ & 0.000 \\
Range & $5.5-11.7$ & $6.5-13.6$ & $7.820 \bullet$ \\
GRBAS scale & & & 0.000 \\
Mean \pm SD & $7.72 \pm 2.88$ & $5.69 \pm 3.00$ & $0-10$ \\
Range & $0-12$ & & \\
\hline
\end{tabular}

Table 4: The improvement in parameters of acoustic analysis pre and post ILSI

\begin{tabular}{lcccc}
\hline Acoustic analysis & & Pre & Post & t \\
\hline Fo & Mean \pm SD & $173.21 \pm 76.33$ & $183.21 \pm 68.09$ & $-2.320 \bullet$ \\
& Range & $90-327.4$ & $109-317.4$ & 0.028 \\
Jitter\% & Mean \pm SD & $2.98 \pm 1.21$ & $2.63 \pm 1.23$ & $-1.994 \neq$ \\
& Range & $1.34-6.1$ & $1.05-5.9$ & $-2.074 \neq$ \\
Shimmer \% & Mean \pm SD & $5.18 \pm 2.55$ & $4.74 \pm 2.68$ & 0.046 \\
& Range & $1.9-13.5$ & $1.3-13.2$ & 0.038 \\
NHR & Mean \pm SD & $0.20 \pm 0.18$ & $0.19 \pm 0.19$ & $-2.232 \neq$ \\
\end{tabular}

The intra judge reliability of the 1 st observer for the size of the lesion, GC, and grade of dysphonia was $93 \%$, $88 \%$, and $90 \%$, respectively, and of the 2 nd observer was $91 \%, 86 \%, 90 \%$, respectively.

The inter-observer agreement showed no significant difference in the grade of dysphonia, lesion size, and GC. Pre ILSI, the values were $91.4 \%, 83.8 \%$, and $92.1 \%$, respectively, and post-ILSI, they were $85.2 \%, 84.6 \%$, and $89.5 \%$, respectively, indicating perfect agreement.

The procedure lasted roughly 15 to 20 minutes. Some patients reported pain and discomfort after the procedure, but it was tolerable, and no medication was prescribed. Following the injection, eight individuals had a mild vocal hematoma that recovered spontaneously during followup. There were no further problems identified, such as VF fibrosis, atrophy, or scarring.

\section{DISCUSSION}

BVFLs, including vocal nodules, polyps, cysts, Reinke's edema, and vocal process granuloma, are typically the result of long-term vocal abuse or misuse. Phonotrauma, chronic inflammation, and subsequent remodeling of the Reinke's space are all typical pathologies ${ }^{[9]}$.

Vocal behavioral modification with systemic steroids and anti-reflux measures is generally the essential first step in treatment. When behavioral measures fail or are impossible for some patients, MLS is usually recommended despite having many adverse effects ${ }^{[14]}$.

Many studies have dealt with office-based ILSI showing the effect of steroids in the treatment of BVFLs. Studies also supported their effects in decreasing collagen deposition and fibroblast proliferation and in reducing extracellular matrix deposition ${ }^{[15]}$.

Some studies used transoral and percutaneous approaches for ILSI as studies done by Tatya et al.(2003), Tatya et al. (2004), Mortensen and Woo, (2006), Hsu et al. (2009) and Woo et al. (2011) ${ }^{[7,8,16-18]}$ but they reported many limitations and low completion rate. In the present study, TESI was done, and the completion rate was high (94.3\%). This could be explained by good tolerance to the transnasal approach over the transoral and percutaneous approaches. 
Also, it can be explained by proper local anesthesia and limited manipulation. The transnasal endoscope enables quick and accurate introduction of topical anesthetics into the laryngeal introitus via the flexible endoscope operating channel. Additionally, the magnified endoscopic field and direct view of the needle tip guarantee that corticosteroids are administered accurately and efficiently into the Reinke's area. Betamethasone showed the same results as dexamethasone used by Wang et al. (2013) with no noticeable complications ${ }^{[19]}$. Betamethasone, like dexamethasone, is preferable in the TESI as it is of low viscosity, so it doesn't cause deposition or clotting through a long and flexible needle.

VLS was done pre-injection and one week, one month, and three months post-injection, but the results were adopted only pre-injection and three months post-injection. VLS was done to assess the size of the lesion and the degree of GC.

VLS evaluation revealed highly significant improvement as $68.9 \%$ showed improvement in the size of the lesion. These results came similar to the results of other studies ${ }^{[5,9,10,16-18,20-22]}$. and demonstrated significant improvement in the size of the lesions after VFSI.

GCis an important stroboscopic finding that was assessed in this study. Post-treatment assessment revealed significant improvement, as $89.6 \%$ of the cases had a mild or no glottal gap post-ILSI. Our results were similar to Mortensen and Woo, (2006) who demonstrated significant improvement regarding the glottal closure after one month of VFSI ${ }^{16]}$. Also, Woo et al. (2011) showed significant improvement in the glottal closure post-VFSI ${ }^{[18]}$.

Regarding the results of the vocal process granuloma, we noted its complete disappearance after four injections, which was similar to the results of a study carried out by Wang et al. (2013) on ten patients with vocal process granuloma, who received two to three injections and reported complete remission of six cases and much improvement in four cases ${ }^{[19]}$.

In comparison to a study done by Ezzat (2019) on the management of recurrent laryngeal granuloma ${ }^{[23]}$, which reported that vocal process granuloma could respond well to conservative treatment with complete recovery in a maximum period of 24 months even it is large and recurrent, ILSI can improve vocal process granuloma in a short time with minimal side effects.

VHI-10 showed a highly significant reduction from 91.38 points before ILSI to 53.45 points post-ILSI. Similar results were found in previous studies ${ }^{[5,9,10,20,22]}$.

The summation of the GRBAS scale showed highly significant improvement in the study, it decreased from $7.72 \pm 2.88$ to $5.69 \pm 3.00$, and the results came similar to results of other researchers ${ }^{[10,16-18,20,21]}$ who reported significant improvement in GRBAS scale. In this study, APA of the degree of dysphonia was particularly assessed and showed highly significant improvement.

To evaluate the accuracy of the results regarding the VLS and the degree of dysphonia, further assessment of the improvement in the two parameters pre and post-treatment was done by two observers in addition to assessing Kappa agreement. There was no significant difference between the two observers.

MPT showed a highly significant improvement mean estimated to be 12.73 seconds post-treatment, compared to 10.24 seconds pre-ILSI. Also, F0, Jitter \% and shimmer $\%$ and NHR showed significant improvement, and these results came similar to results of other previous studies $^{[9,10,17,18,20,22,24]}$.

The minimal discrepancy which may be present between the results of this study and the results of previous studies may be due to the difference in the types of the lesions injected, sample size, and the follow-up periods and this indicates that the reduction in the size of BVFLs is owing to corticosteroid injection regardless of the used technique and the injectable material and the choice of the technique depends on surgical experience, technical support, and tolerability of the patient.

\section{LIMITATION OF THE STUDY}

The stroboscopic evaluation was omitted from this study due to the variety of the lesions observed, which made meaningful comparisons of changes in appearance impossible. Additionally, due to the limited sample size, further statistical analysis of subgroups was difficult. Also, we couldn't assess the recurrence rate due to short term follow-up period, and the lack of a control group didn't give us the chance to compare other treatment options as MLS and voice therapy.

\section{CONCLUSION}

Office-based VFSI is an effective alternative treatment option in all types of BVFLs. However, posterior granuloma might need more than one injection to resolve. Betamethasone can be used as a safe and reliable replacement for dexamethasone. Office-based TESI is a more practical and less technical procedure. Further studies are needed to compare the outcome of MLS under direct laryngoscope versus office-based steroid injection combined with good voice hygiene and voice therapy.

\section{CONFLICT OF INTEREST}

There are no conflicts of interest. 


\section{REFERENCES}

1. Ramavat A, Tiwana H, Banumathy N, Bakshi J, Panda $\mathrm{N}$ and Goel A. Efficacy of Intralesional Steroid Injection in Small BVFLs. Journal of Voice. 2018; 33. 10.

2. Baraka M, El-Dessouky H, Behiry E, Ezzat E, ElAshry R. Role of steroids injection in treatment of minimal associated pathological lesions. Menoufia Medical Journal. 2020; 33(2):333-338.

3. Chitguppi C, Anoop R, Meher R, Rathore PK. Is the voice of a professional voice user with no vocal cords lesions similar to that of non-professional voice users? J of Voice. 2017;33(1):66-72.

4. Graupp M, Bachna-Rotter S, Gerstenberger C, Friedrich G, Fröhlich-Sorger E, Kiesler K, Gugatschka $M$. The unsolved chapter of vocal fold scars and how tissue engineering could help us solve the problem. European Archives of Oto-Rhino-Laryngology. 2016; 273(9):2279-84

5. Al-Ali M, Anderson J. The role of steroid injection for vocal folds lesions in professional voice users. J of Otolaryngol - Head and Neck Surg. 2020; 49(50).

6. Campagnolo A, Tsuji D, Sennes L, Imamura R. Steroid injection in chronic inflammatory vocal fold disorders, literature review. Braz J Otorhinolaryngol 2008; 74:926-932.

7. Tateya I, Kojima H, Hirano S, Kaneko K, Omori K and Ito J. Steroid injection for RE using fiberoptic laryngeal surgery. Acta Otolaryngol. 2003; 123: $417-420$.

8. Tateya I, Omori K, Kojima H, Hirano S, Kaneko K, Ito J. Steroid injection to vocal nodules using fiberoptic laryngeal surgery under topical anesthesia. Eur Arch Otorhinolaryngol. 2004; $261: 489-492$.

9. Abdelgelil AS, Dawood YM, Ibraheem MK. Transnasal endoscopic steroid injection for treatment of vocal fold polyps (a noninvasive technique). AlAzhar Assiut Med J. 2018; 16:241-6.

10. Wang C, Lai M, Liao L, Lo W and Cheng P. Transnasal endoscopic steroid injection: practical and effective alternative treatments for benign VF disorders. Laryngoscope. 2013; 123: 1464-1468.

11. Wang C, Liao L, Cheng $\mathrm{P}$, Lo $\mathrm{W}$ and Lai $\mathrm{M}$. Intralesional steroid injection for benign VF disorders: a systematic review and meta-analysis. Laryngoscope. 2013; 123:197-203.

12. Kotby MN, Barakah M, Abou El Ella MY, El Sady S, El Shobary A and Refaie N. Introduction to vocology. In Kotby MN (ed.), Clinical Vocology. Ain Shams, 2016.

13. Zubčić Ž, Mendeš T, Včeva A, Mihalj H, Bogović V, Milanković S. Presence of pepsin in laryngeal tissue and saliva in benign and malignant neoplasms. Biosci Rep. 2020;40(11).

14. Dassé R, Pujol E. First-line treatment of exudative vocal fold-lesions by in-office local corticosteroid injection: A literature review. European Annals of Otorhinolaryngology, Head and Neck Diseases. 2020; in press.

15. Campagnolo, A. M., Tsuji, D. H., Sennes, L. U., Imamura, R. and Saldiva, P. Histologic study of acute vocal fold wound healing after corticosteroid injection in a rabbit model. Annals of Otology, Rhinology and Laryngology 119, 133-139 (2010).

16. Mortensen M, Woo P. Office steroid injections of the larynx. Laryngoscope 2006; 116:1735-1739.

17. Hsu Y, Lan M, Chang S. Percutaneous corticosteroid injection for vocal fold polyp. Arch Otolaryngol Head Neck Surg. 2009; 135:776-780.

18. Woo JH, Kim DY, Kim JW, Oh EA, Lee SW. Efficacy of percutaneous vocal fold injections for benign laryngeal lesions: Prospective multicenter study. Acta Otolaryngol. 2011; 131 :1326-1332.

19. Wang C, Lai MS, Lo W, Liao L and Cheng P. Intralesional steroid injection: an alternative treatment option for vocal process granuloma in ten patients: Clinical Otolaryngology. 2013; 123:197-203.

20. Wang C, Lai M and Hsiao T. Comprehensive Outcome Researches of Intralesional Steroid Injection on BVFLs. J Voice. 2015; 29(5):578-587.

21. Wang C, Lai M and Cheng P. long term surveillance following intralesional steroid injection for BVFLs. Otolaryngology, Head and Neck Surgery. 2017; 124: $510-515$.

22. Lee S, Park K. Long-term efficacy of percutaneous steroid injection fortreating benign vocal fold lesions: a prospective study: percutaneous steroidinjection. Laryngoscope. 2016;126(10):2315-9.

23. Ezzat E. The new in management of recurrent laryngeal granuloma: long term follow-up. J Otolaryngol ENT Res. 2019;11(5):245-251.

24. 24. Lee S, Yeo J, Choi J, Jin HJ, Kim JP, Woo $\mathrm{SH}$, Jin SM. Local steroid injection via the cricothyroid membrane in patients with a vocal nodule. Arch Otolaryngol Head Neck Surg 2011; 137:1011-1016 